\title{
Desempenho de Controladores IMC Aplicados na Perfuração de Poços de Petróleo em reservatórios offshore
}

\section{Performance of IMC controllers applied to oil well drilling}

\author{
Carlos Alvarado', Victor Gamarra * \\ 1 Universidad Señor de Sipán, Facultad de Ingeniería, Arquitectura y Urbanismo, Perú. \\ 2 Universidade Estadual Paulista “Julho de Mesquita Filho”, Faculdade de Engenharia de Guaratinguetá, Brasil.
}

Recibido (Received): 14/10/2018

Aceptado (Accepted): 15/12/2018

\begin{abstract}
RESUMO
O controle de pressão durante a perfuração de poços de petróleo pode ser um dos processos mais complexos e perigosos do estágio da exploração. Este estudo propõe o desenho e a validação do desempenho dos Controladores de Modelo Interno (IMC e SIMC) para controlar a pressão na perfuração de poços de petróleo com base na técnica Manage Pressure Drilling (MPD). O MPD adiciona uma válvula de controle no sistema de perfuração para obter uma outra variável de manipulação na pressão no fundo do poço. Na primeira parte deste trabalho, obteve-se um modelo matemático linear do processo, fundamentado na mecânica dos fluidos. O processo dinâmico apresentou um elemento integrador, além disso, é considerado o acréscimo de um intervalo de tempo entre a ação da válvula e a resposta da variação da pressão no fundo do poço. Na segunda parte, o controlador IMC melhorado foi projetado para compensar o efeito do termo integrado com o atraso de tempo, procurando o melhor desempenho e robustez do sistema. Finalmente, o controlador proposto é testado por problemas comuns durante simulações de perfuração (perda de fluido, influxos, conexão de tubulação e perda de potência da bomba) utilizando data de um caso de reservatorio offshore, mostrando sua viabilidade. Além disso, o desempenho do sistema de malha fechada é comparado com um controlador PI clássico.
\end{abstract}

Palabras Clave: Controlador IMC-PID, Control de Pressão, Manage Pressure Drilling, Perfuração de Poços de Petróleo

\section{ABSTRACT}

Pressure control during drilling of oil wells can be one of the most complex and dangerous processes of exploration stage. This study proposes the design and validation of the performance of Internal Model Controllers (IMC and SIMC) to control the pressure in the drilling of oil wells based on technical Manage Pressure Drilling (MPD). MPD adds a control valve in the drilling system to have another manipulate variable on the well pressure. In the first part of this work, there was obtained a mathematical linear model of the process, which is founded on fluid mechanics. The dynamic process showed an integrator element, moreover, it is considered the addition of a time-delay between the action of valve and the response of the downhole pressure variation. In the second part, the improved IMC controller was designed to offset the effect of the integrate term with time delay looking for the best performance and robustness of the system. Finally, the proposed controller is tested by common problems during drilling simulations (loss of fluid, influxes, pipe connection and loss of pump power) using data from a case of offshore reservoir, showing its viability. In addition, the performance of the closed loop system is compared with a classical PI controller.

Keywords: IMC-PID controller, Pressure control, Manage pressure drilling, Oil wells drilling.

\section{INTRODUCCION}

Nas ultimas três décadas, o consumo de energia de fontes renováveis incrementou-se num $4.9 \%$ no mundo todo; contudo, o petróleo continua aportando a maior porcentagem do total da energia que se consume. Este ainda é um enorme negócio no qual se investem centenas de bilhões de dólares em exploração cada ano [1].

$\mathrm{Na}$ atualidade, as indústrias petroleiras para a obtenção do petróleo primário dividem o processo de exploração em três etapas: a prospecção, perfuração e a extração. A prospecção é a localização de bacias sedimentares por meio de análise detalhada do solo e subsolo. Depois de localizado o local onde provavelmente haverá uma reserva de petróleo, é feita a perfuração que é a criação de um meio de conexão entre as jazidas e a superfície normalmente chamada de poço de perfuração. Quando é realizada a primeira perfuração e se obtém sucesso, são feitas outras

\footnotetext{
* Corresponding author.:

E-mail: carlosalvaradosilva@gmail.com
} 
perfurações para saber a extensão da bacia petrolífera e ver se é realmente viável realizar o próximo passo: a extração. A extração é o processo de levar o combustível fóssil à superfície [2] - [4].

Durante a exploração de poços de petróleo podemse criar uns dos mais terríveis cenários originando, grandes perdas econômicas, danos ambientais e até perdas humanas como foram os acidentes ocorridos na plataforma de Piper Alpha (no Mar do Norte, em 1988), na Plataforma de Enchova (Bacia de Campos, RJ) em 1984 e 1988, entre outros. Todos estes acidentes simbolizam o grande potencial de perigo que existe nas plataformas de petróleo [5].

Das três etapas mencionadas, a perfuração, geralmente, é a fase mais custosa, difícil e perigosa da exploração de petróleo [6]. A perfuração consiste na aplicação de uma força sobre a sonda de perfuração que é composta por uma torre metálica que sustenta a coluna de perfuração com uma broca na sua extremidade. A coluna de perfuração é composta por tubos de 9 metros de comprimento que são conectados entre si [2].

Nas operações de perfuração, precisa-se da circulação de um fluido com a finalidade de retirar os restos rochosos próprios da perfuração. Este fluido é chamado lama e é bombeada pela coluna de perfuração que flui através da broca no fundo do poço. Esta lama atua como esfriador da broca, transporta restos rochosos (cuttings) para a saída do poço, e também adiciona uma pressão hidrodinâmica no fundo do poço [7].

Controlar a pressão no fundo do poço (CPFP) é de vital importância durante a perfuração para manter a seguridade total do processo. A perfuração convencional fica limitada por duas pressões: A pressão hidrostática (própria da profundidade) e hidrodinâmica (gerada pela bomba principal de circulação de fluido). Em quanto, a nova tecnologia de perfuração (MPD Manage Pressure Drilling) utiliza a técnica de manter a pressão controlada no fundo do poço adicionando uma pressão de controle além da pressão hidrodinâmica e a pressão hidrostática. Esta nova pressão é produzida pela manipulação da válvula de estrangulamento na saída do poço [8].

Normalmente, nas plataformas marítimas (offshore), um operador ajusta manualmente o motor de arranque da válvula, em operações MPD manuais. A válvula deve ser fechada para reduzir o aumento de pressão no fundo no caso seja excessiva. Na verdade, é uma tarefa difícil devido à rápida flutuação da pressão. No entanto, na operação de perfuração manual, a possibilidade de erros é bastante elevada, bem como há limitações sobre o tempo de resposta. Por tanto, é necessário a utilização de um sistema automatizado para manter a pressão no fundo da maneira desejada [4].

Além do mais, o processo da perfuração torna-se ainda mais complicado devido à geologia da região a perfurar. Em todo terreno, existem certos limites de pressão que devem ser tomados em consideração, pressões próprias e dependentes da geologia; principalmente dois: a pressão do poro e a pressão de fratura (perfis de pressão). Todas as formações são porosas até certo ponto, estes poros podem conter água, gás, óleo ou uma combinação destas. A pressão exercida pelos fluidos dentro destes poros é chamada "pressão de poro" [9].

A quantidade de pressão que a formação pode suportar antes de falhar é conhecida como a pressão de fratura. Ela pode também ser definida como a pressão à qual a formação se fratura e o fluido circulante é perdido. Assim, a pressão deve ser mantida entre estes limites o que gera a necessidade de um controle com resposta rápida e precisa às mudanças da pressão no poço para o sucesso da perfuração [2], [4].

As estratégias de controle são comuns na indústria e fornecem redução de custos e confiabilidade para diferentes processos. Embora o sistema de controle moderno seja uma realidade em muitas áreas industriais (refinarias, petroquímicas e plantas de gás), não é amplamente utilizado para unidades de perfuração de petróleo. A automação para a tecnologia de construção de poços ainda é um conceito emergente, em discussão dentro da comunidade técnica. Kennett e Smith [10] são pioneiros em apresentar a discussão sobre automação para a indústria de perfuração e listar as principais preocupações para implementação no terreno: a complexidade do processo, confiabilidade, etc [11].

Entre as mais atraentes técnicas de controle de pressão com retroalimentação está o controlador PID (Proporcional, Integral e Derivativo), devido à simplicidade da sua estrutura e à fácil implementação no meio industrial. Entretanto, sabe-se da teoria que este controlador precisa de uma boa sintonia dos seus parâmetros para termos boa resposta do sistema, isto quando o modelo é linear, invariante no tempo e sem tempo de atraso, o que não ocorre na pratica [12], [13].

$\mathrm{Na}$ atualidade, os tipos de controle em sistemas de perfuração estão sendo estudados consideravelmente devido aos benefícios que implicariam o ótimo controle do desenvolvimento do sistema global, não só em custos econômicos, mas também avanços de seguridade, e sustentabilidade. [7], [13] - [15]. Nesses estudos, os sistemas foram modelados com funções de transferência de primeira ordem e sem considerar o tempo de atraso, com a metodologia de controle do tipo IMC (Internal Model Controller).

Neste estudo, a diferença com os outros trabalhos está no modelo do processo real, o qual é dado por uma função de transferência de primeira ordem, adicionado de um termo integrativo. Assim como, adicionasse um tempo de atraso no processo de perfuração do poço, parâmetro bem mais próximo da realidade considerando-se o instante da aquisição dos dados reais e a ação do controle.

A fim de determinar e especificar qual o tipo de controle que leva o sistema a ter as melhores 
características e melhor desempenho, compara-se e avalia-se o desempenho de três tipos de controles de pressão. Obtêm-se os resultados da simulação de dois controles com a metodologia tipo IMC e SIMC (Simple IMC), respectivamente, e os resultados de um controlador PI clássico, aplicados na perfuração de poços de petróleo.

Em aplicações de controle industrial, mesmo com variações de referências na operação do processo, uma boa resposta transitória também é desejada. Além da obtenção da variável desejada é importante que o sistema de controle também elimine distúrbios elou ruídos inerentes do processo como: Conexões de tubos, influxos, perda de lama, etc. Assim, em processos integrativos, esta exigência se acentua devido ao termo integrativo no modelo, o qual pode causar uma resposta lenta na presença de distúrbios.

\section{MODELAGEM DO SISTEMA}

Nesta secção, apresenta-se o modelo matemático do sistema que trata do controle de pressão na perfuração de poços petrolíferos. Os fundamentos teóricos utilizados são da mecânica dos fluidos, principalmente, a equação de transporte de Reynolds para a obtenção das equações diferenciais que tratam da pressão no fundo do poço e da vazão da válvula de controle. No sistema de perfuração de poços, o fluido de perfuração (ou lama) percorre pelo interior dos tubos de perfuração para a broca e circula pela zona anular levando os restos rochosos da perfuração até a superfície do poço, passando pela válvula de estrangulamento e voltando para o deposito de lama, fechando o sistema, conforme se ilustra na Fig.1.

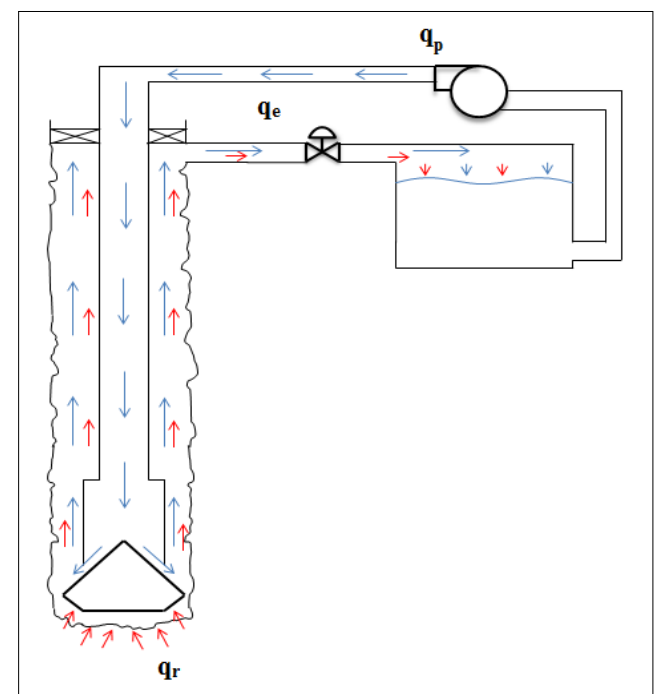

Figura 1 Un Esquema do sistema de perfuração de poços.

As equações resultantes que descrevem o modelo do sistema, e a definição das variáveis (Tabela 1), estão a seguir [16],

$$
\begin{aligned}
V_{c} \dot{P}_{p}= & \beta_{c}\left(q_{p}-q_{b}\right) \\
V_{a} \dot{P}_{e}= & \beta_{a}\left(q_{b}+q_{r}+q_{c}-q_{e}\right) \\
\dot{q}_{b} M= & P_{p}-P_{e}-F_{a}\left(q_{b}+q_{r}\right)^{2}-F_{c} q_{b}{ }^{2}+\left(\rho_{c}-\rho_{a}\right) g h_{b} \\
P_{b} M= & M_{c} P_{e}+M_{a} P_{p}+M_{c} F_{a}\left(q_{b}+q_{r}\right)^{2}-M_{a} F_{c} q_{b}{ }^{2} \\
& +\left(M_{a} \rho_{c}-M_{c} \rho_{a}\right) g h_{b}
\end{aligned}
$$

TABELA 1 NOMENCLATURA.

\begin{tabular}{ll}
\hline$V_{a}$ & Volume da região anular \\
$V_{c}$ & Volume da região da coluna de perfuração \\
$\mathrm{P}_{\mathrm{p}}$ & Pressão da bomba principal \\
$\mathrm{P}_{\mathrm{e}}$ & Pressão da válvula de estrangulamento \\
$\mathrm{P}_{\mathrm{b}}$ & Pressão na broca \\
$\mathrm{q}_{\mathrm{e}}$ & Vazão da válvula de estrangulamento \\
$\mathrm{q}_{\mathrm{b}}$ & Vazão da saída da broca \\
$\mathrm{q}_{\mathrm{p}}$ & Vazão da bomba principal \\
$\mathrm{q}_{\mathrm{r}}$ & Vazão do reservatório para o poço \\
$\beta_{\mathrm{a}}$ & Modulo de compressibilidade da região anular \\
$\beta_{\mathrm{c}}$ & Modulo de compressibilidade da região da coluna \\
$\mathrm{q}_{\mathrm{c}}$ & Vazão da bomba de contra pressão \\
$\mathrm{M}_{\mathrm{a}}$ & Massa da região anular \\
$\mathrm{M}_{\mathrm{c}}$ & Massa da região da coluna de perfuração \\
$\mathrm{M}$ & Soma de Ma e Mc \\
$\rho_{a}$ & Densidade da região anular \\
$\rho_{c}$ & Densidade da região da coluna de perfuração \\
$\mathrm{h}_{\mathrm{b}}$ & Profundidade da broca \\
$\mathrm{g}$ & Gravidade \\
$\mathrm{F}_{\mathrm{a}}$ & Força de atrito da região anular \\
$\mathrm{F}_{\mathrm{c}}$ & Força de atrito da região da coluna de perfuração \\
\hline
\end{tabular}

Para efeitos de estudo das metodologias de controle, o modelo deve ser linearizado. Para isto se utiliza a série de Taylor e lineariza-se o modelo em torno do ponto de operação [17]. Primeiro, se identificam as variáveis de entrada e saída do sistema. Entre as variáveis de entrada foram consideradas: $q_{p}, q_{r}, q_{c}$, qe e $h_{b}$. E considera-se a entrada $u$ como sendo a soma da vazão da válvula de estrangulamento e a vazão produzida pela bomba de contra pressão $\left(u=q_{c}-q_{e}\right)$.

Assim, a equação de estado do sistema linearizado é dada pela relação das variáveis de entrada (U), as variáveis de estado $(\mathrm{X})$ e as variáveis da saída (Y), conforme a seguir,

$$
\begin{aligned}
& \left.\dot{X}=J_{a} X+J_{b} U\right), x(0)=x_{o} \\
& Y=J_{c} X+J_{d} U
\end{aligned}
$$

Contudo, para projetar os controladores deve-se modelar o processo em função de uma estrutura predeterminada. Assim, a função de transferência que melhor se aproxima ao modelo linear é uma equação de primeira ordem, com elemento integrativo, caracterizado pelos parâmetros: "K" e " $\tau$ ", como se mostra a seguir, 


$$
\frac{P_{b}(s)}{u(s)}=\frac{K}{s(\tau s+1)}
$$

\section{CONTROLE IMC}

Destaca-se que estas duas metodologias (IMC e SIMC) são utilizadas na sintonização dos parâmetros do controlador PID. A metodologia de controle tipo IMC (tradicional) providencia uma estrutura adequada com informação que auxilia na sintonização do controlador, com maior simplicidade, melhorando o desempenho e robustez do sistema [18]. A Fig. 2(a) mostra a estrutura do controlador IMC que é dada pelo processo real $G(\mathrm{~s})$, o modelo do processo $\bar{G}(s)$ e a função de transferência $q(s)$. A função $q(s)$ é a única expressão manipulável e que pode mudar a resposta do sistema realimentado. $O$ sinal de saída do $\mathrm{q}(\mathrm{s})$ afeta a $\mathrm{G}(\mathrm{s})$ e $\bar{G}(s)$, e a diferença entre a saída de estes sinais é restado do sinal de referência, o que gera o erro de retroalimentação.

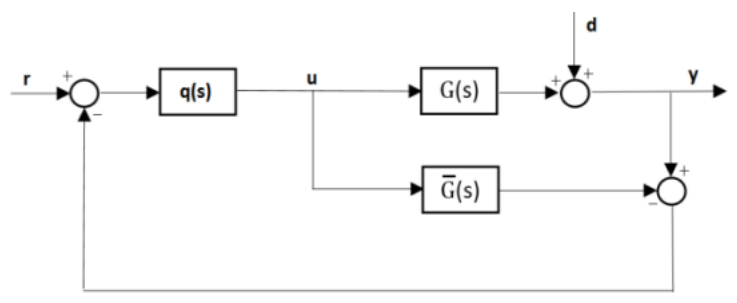

(a)

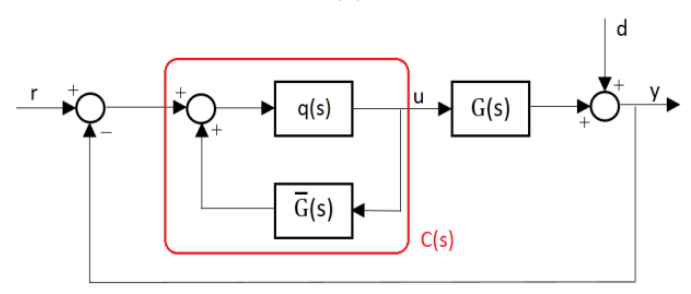

(b)

Figura 2. (a) Estrutura de um sistema com controlador IMC (b) Estrutura retroalimentada genérica.

Entretanto, fazendo um arranjo de diagrama de blocos obtém-se uma nova estrutura, conforme a Fig. 2(b). Obtendo a função de transferência do controlador $C(s)$, dada pela relação entre $\mathrm{q}(\mathrm{s})$ e $\bar{G}(s)$, tornando-se uma estrutura de retroalimentação genérica. Assim, da relação entre a referência, o distúrbio e a saída do sistema tem-se,

$$
\begin{aligned}
& y=\frac{q G}{1+q(G-\bar{G})} r+\frac{1-q \bar{G}}{1+q(G-\bar{G})} d \\
& y=\eta r+\varepsilon d
\end{aligned}
$$

sendo, $\eta$ a sensibilidade complementar e $\varepsilon$ a função da sensibilidade. No diagrama de bode, a função de sensibilidade informa sobre a robustez do sistema. Quando o modelo e a planta coincidem $(G=\bar{G})$ as expressões $\eta$ e $\varepsilon$ se simplificam, isto é,

$$
\begin{aligned}
& \bar{\eta}=q \bar{G} \\
& \bar{\varepsilon}=1-q \bar{G}=1-\bar{\eta}
\end{aligned}
$$

Também, para garantir que o sistema não apresente problemas de offset devido a mudanças de referência ou distúrbios no processo, deve-se cumprir:

Para entradas degrau (tipo 1):

$\lim _{s \rightarrow 0} q \bar{G}=\bar{\eta}(0)=1$

Para entradas rampa (tipo 2):

$\lim _{s \rightarrow 0} \frac{d}{d s}(q \bar{G})=\left.\frac{d \bar{\eta}}{d s}\right|_{s=0}=0$

O comportamento ideal do sistema se apresenta quando y é igual a $r$ (Eq. 8), assim, deve-se ter $\eta=1$ e $\varepsilon=0$. Para isto, procura-se um q, que seja o inverso do modelo do processo $\bar{G}$ para garantir a igualdade. E se verifica, que o sistema retroalimentado, com controlador, é estável se apenas $\mathrm{G}$ e q são estáveis.

Destaca-se que um processo com elemento integrativo é instável por natureza. Para este caso, a entrada ao degrau unitário em conjunto com o elemento integrativo do processo gera uma função rampa. Assim, para eliminar o problema de offset o requisito exigido pela Eq. (12) se torna necessário.

Por outro lado, há requerimentos que a função q deve cumprir para garantir a capacidade e bom desempenho do controlador IMC. Um deles é que os polos devem-se encontrar no lado esquerdo do plano s. E um segundo requerimento, devido à derivação da entrada degrau retroalimentada, a que gera impulsos infinitesimais no tempo, e de difícil implementação [17]. Assim, a fim de evitar a diferenciação pura destes sinais deve-se garantir que a função q seja Própria, isto é,

$$
\lim _{s \rightarrow \infty}|q| \geq 0
$$

E um último requerimento denomina-se causalidade. A função q deve ser causal, o que significa que o controlador não deve exigir predição, ou seja, ele deve levar em conta somente as medições atuais e anteriores da planta. Um exemplo simples de uma função de transferência não causal é o inverso de uma função de transferência do tempo de atraso. 
Assim, a especificação do IMC é dada conforme a seguir: Primeiro, garantir a estabilidade e causalidade de q; para isso, o modelo do processo se divide em duas partes, a primeira contém todos os elementos que são de fase não-mínima $\bar{G}_{-}$e a outra, de fase mínima $\bar{G}_{+}$, $\left(G=\bar{G}_{-} \bar{G}_{+}\right)$. Desta forma, pode-se denominar o inverso da fase não-mínima como $\bar{q}=\bar{G}_{-}-1$ o que satisfaz os requisitos.

A outra parte, a fase mínima, pode ser fatorada conforme se define a seguir,

$$
\bar{G}_{+}=e^{-\theta} \prod_{i}\left(-B_{i} s+1\right) ; \operatorname{Re}\left(B_{i}\right)>0
$$

ou

$$
\bar{G}_{+}=e^{-\theta} \prod_{i}\left(\frac{-B_{i} s+1}{B_{i} s+1}\right) ; \operatorname{Re}\left(B_{i}\right)>0
$$

sendo, $\theta$ o tempo de atraso e $B$ a constante de tempo. Estas expressões descrevem o modelo com polos do lado direito do plano s. A escolha de uma de estas equações, (14) ou (15), depende dos índices de erro, IAE (Integral absolute error) e ISE (Integral square error), respetivamente.

Segundo deve-se exigir que a função q seja Própria, ou seja, satisfazer com a condição dada pela Eq. (13). De (9) obtém-se a relação $\bar{\eta}=q \bar{G}_{-} \bar{G}_{+}$. É importante distinguir que q não é igual que $\bar{q}$. A função $\bar{q}$ cancela os valores de $\bar{G}_{-}$, além disso, $\bar{q}$ é não própria. Então, para garantir o último requisito deve-se adicionar um filtro $\mathrm{f}(\mathrm{s})$ à função $\bar{q}$. Assim, fazendo $q=\bar{q} f(s)$ obtém-se,

$$
\bar{\eta}=\bar{q} f(s) \overline{G_{-}} \bar{G}_{+}
$$

Uma estrutura de filtro comum que elimina o offset

\begin{tabular}{llll}
\hline Controle & $\mathrm{K}_{\mathrm{p}}$ & $\mathrm{K}_{\mathrm{I}}$ & $\mathrm{K}_{\mathrm{D}}$ \\
\hline IMC & $\frac{\theta+2 \lambda+\tau}{(\theta+\lambda)^{2}}$ & $\theta+2 \lambda+\tau$ & $\frac{\tau(\theta+2 \lambda)}{\theta+2 \lambda+\tau}$ \\
SIMC & $\frac{1}{K}\left(\frac{1}{\tau_{c}+\theta}\right)$ & $4\left(\tau_{c}+\theta\right)$ & $\tau$ \\
\hline
\end{tabular}

para entradas degrau ou rampas é dada por,

$$
f(s)=\frac{1}{(\lambda s+1)^{n}}
$$

A ordem do denominador $\mathrm{n}$ é escolhida de forma a satisfazer a Eq. (13) o que garante a função q como sendo própria. O parâmetro $\lambda$ é ajustável e determina a velocidade de resposta e a robustez do sistema.

Para casos em que o processo apresenta um elemento integrativo, como já foi explicado, deve-se considerar a condição (12). Assim, de (16) e (12) obtém-se,

$$
\left.\frac{d}{d s}\left(\bar{q} f(s) \bar{G}_{-} \bar{G}_{+}\right)\right|_{s=0}=\left.\frac{d}{d s}\left(f(s) \bar{G}_{+}\right)\right|_{s=0}=0
$$

E, para satisfazer a Eq. (18) o filtro $f(s)$ deve ser dado por,

$$
f(s)=\frac{\left(2 \lambda-\bar{G}_{+}(0)\right) s+1}{(\lambda s+1)^{n}}
$$

sendo $\bar{G}_{+}(0)$ a fase mínima do modelo quando s tende a zero.

Um outro controlador mais simples mantendo a mesma estrutura do controlador IMC tradicional, denominado SIMC (Simple Internal Model Controller), tem sido desenvolvido por Skogestad [19]. O qual consiste em especificar a resposta desejada do sistema realimentado e realizar os cálculos analíticos dos parâmetros para obter essa resposta. Para isto, ajustasse o sistema realimentado (processo mais controlador) para uma função de transferência de primeira ordem desejada com tempo de atraso, o qual é o mesmo tempo de atraso do processo, conforme a seguir,

$$
\left[\frac{y(s)}{r(s)}\right]_{d e s}=\frac{K}{\tau_{c} s+1} e^{-\theta s}
$$

sendo, $\tau_{\mathrm{c}}$ o parâmetro do processo de primeira ordem, Ko mesmo parâmetro da Eq. (6), e $\theta$ o tempo de atraso. Fazendo desta forma, com que a resposta do sistema se comporte de maneira desejada (sobressinal, tempo de resposta, tempo de estabilização, etc.).

E finalmente, mediante métodos algébricos obtémse analíticamente os parâmetros do controlador PID para um processo integrativo com tempo de atraso, obtidos pelas metodologias IMC e SIMC, respectivamente, e apresentadas na Tabela 2.

TABELA 2 PARÂMETROS DO PID PARA UM PROCESSO INTEGRATIVO COM TEMPO DE ATRASO.

\section{ESPECIFICAÇÕES DOS CONTROLADORES}

Projetar e especificar um controlador é uma tarefa difícil devido à instabilidade que se apresentam em processos, principalmente pela presença do integrador no modelo. A característica do processo integrativo é que não consegue atingir a estabilidade em tempo nenhum quando é excitado por uma função degrau unitário em malha aberta. Fisicamente, um processo integrativo ocorre, por exemplo, em sistemas realimentados no controle de nível, pressão e/ou temperatura [20].

Portanto, inicia-se descrevendo o processo real do sistema. E para isto, utilizam-se dados de um estudo de 
caso desenvolvido num reservatório offshore da regiao nordica conforme a Tabela 3, os quais também foram usados em outras pesquisas [14], [15], [21].

TABELA 3 PARÂMETROS CONSTANTES DO SISTEMA DE PERFURAÇÃO.

\begin{tabular}{ccc}
\hline Parâmetros & Valor & Unidades \\
\hline$\beta_{a}$ & 7000 & Bar \\
$\beta_{c}$ & 11000 & Bar \\
$F_{a}$ & 15831 & $10^{-5}\left(\mathrm{~m}^{7} / \mathrm{Kg}\right)$ \\
$\mathrm{F}_{\mathrm{c}}$ & 176640 & $10^{-5}\left(\mathrm{~m}^{7} / \mathrm{Kg}\right)$ \\
$\mathrm{M}_{\mathrm{a}}$ & 1600 & $10^{-5}\left(\mathrm{Kg} / \mathrm{m}^{4}\right)$ \\
$\mathrm{M}_{\mathrm{c}}$ & 5720 & $10^{5}\left(\mathrm{Kg} / \mathrm{m}^{4}\right)$ \\
$\rho_{\mathrm{a}}$ & 0,0119 & $10^{-5}\left(\mathrm{Kg} / \mathrm{m}^{3}\right)$ \\
$\rho_{\mathrm{c}}$ & 0,0125 & $10^{-5}\left(\mathrm{Kg} / \mathrm{m}^{3}\right)$ \\
$\mathrm{V}_{\mathrm{a}}$ & 96,1327 & $\mathrm{~m}^{3}$ \\
$\mathrm{~V}_{\mathrm{c}}$ & 28,2743 & $\mathrm{~m}^{3}$ \\
$\mathrm{q}_{\mathrm{p}}$ & 0,015 & $\mathrm{~m}^{3} / \mathrm{s}$ \\
$\mathrm{q}_{\mathrm{r}}$ & 0,001 & $\mathrm{~m}^{3} / \mathrm{s}$ \\
$\mathrm{h}_{\mathrm{b}}$ & 2000 & $\mathrm{~m}$ \\
\hline
\end{tabular}

Após a substituição das constantes no modelo (Eq. 6), e visto que são instantes diferentes entre o estado atual da pressão de estrangulamento e a ação corretiva, utiliza-se a função de transferência a seguir,

$$
\frac{P_{b}(s)}{u(s)}=\frac{62.22}{s(0.008 s+1)} e^{-2 s}
$$

o qual considera, o efeito que se produz devido ao tempo de atraso. Experimentalmente e segundo a bibliografia [7], o tempo deve ser de aproximadamente 2 seg.

Para sintonizar e especificar os parâmetros PID conforme as metodologias propostas (IMC e SIMC), primeiro deve-se medir o desempenho da resposta transitória. Assim, em cada caso, se simulou no instante inicial uma função degrau unitário como sendo a entrada de referência e, além disso, no instante igual a 100 seg. se adicionou um distúrbio de carga $d(t)$, conforme a Fig. 2(a). O distúrbio foi representado por uma função degrau de valor 0.01. O qual foi utilizado somente para quantificar em ambas metodologias, a rejeição ao distúrbio obtendo-se o valor da variável IAEd, que consiste na integração do erro absoluto do sinal da resposta somente relacionado ao distúrbio.

A seguir, apresentam-se para ambas metodologias os desempenhos quantificados das simulações do sistema, e respectivos parâmetros.
Para efeitos da especificação do controlador PID utiliza-se nesta seção a metodologia IMC. Assim, para diferentes $\lambda$ obtém-se resultados com diferentes comportamentos. Uma primeira observação da gráfica mostra que existe um comportamento indireto entre a resposta transitória e a resposta devido ao distúrbio. Isto quer dizer, que quanto melhor desempenho exista no transitório, o sistema não responderá corretamente na presença de distúrbios, o qual é muito comum em controladores PID. O mesmo acontece com a robustez (Ms) do sistema. É relevante entender que quanto menor o valor do Ms maior a robustez do sistema controlado. A Fig. 3 e a Tabela 4 mostram que a robustez melhora quando maior são os valores de $\lambda$, e a rejeição ao distúrbio $\left(I A E_{d}\right)$ aumenta com o aumento de $\lambda$.

TABELA 4 DESEMPENHO QUANTIFICADO DO SISTEMA E OBTENÇÃO DOS PARÂMETROS PID PARA DIFERENTES VALORES DE $\lambda$.

\begin{tabular}{llllll}
\hline$\lambda$ & $M s$ & $\mathrm{IAE}_{d}$ & $\mathrm{~K}_{\mathrm{P}}$ & $\mathrm{K}_{\mathrm{I}}$ & $\mathrm{K}_{\mathrm{D}}$ \\
\hline 2,5 & 3,38 & 15,8 & 0,0056 & 7,008 & 0,008 \\
3,5 & 2,47 & 18,9 & 0,0048 & 9,008 & 0,008 \\
4,5 & 2,07 & 26,4 & 0,0042 & 11,008 & 0,008 \\
5,5 & 1,81 & 35,1 & 0,0037 & 13,008 & 0,008 \\
6,5 & 1,70 & 45,0 & 0,0033 & 15,008 & 0,008 \\
\hline
\end{tabular}

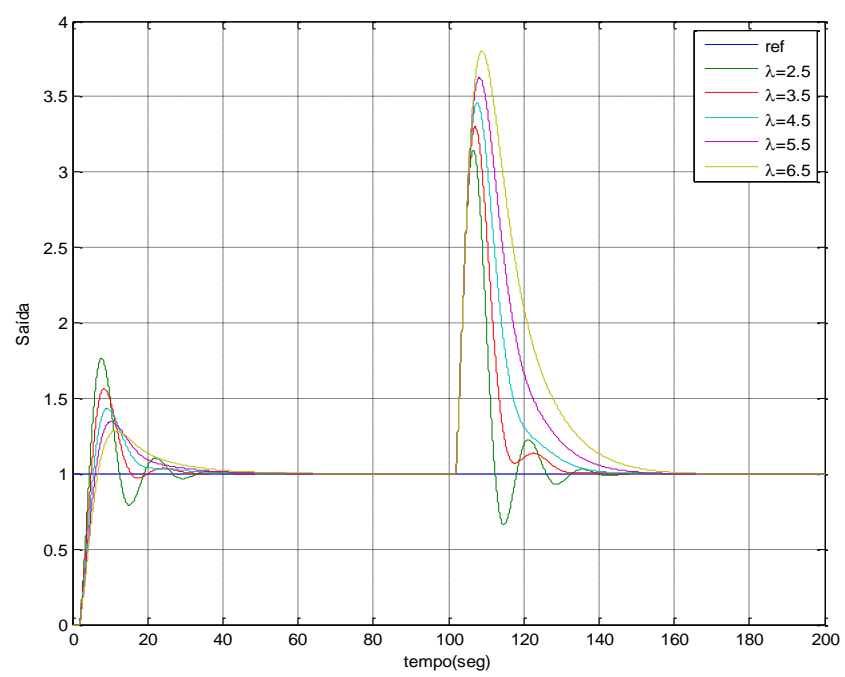

Figura 3. Desempenho do sistema para valores de $\lambda$.

\section{B. Controlador SIMC}

O projeto do controlador PID especificado pela metodologia SIMC apresenta as mesmas tendências entre a resposta transitória, a robustez, a rejeição aos distúrbios, e o parâmetro do modelo interno $\tau$ c. No trabalho de Skogestad [19] obtém-se o parâmetro ótimo đc o qual direciona para a melhor relação entre a resposta transitória e os parâmetros, Ms e IEAd, respectivamente. Isto acontece, quando $\tau_{c}$ é igual ao tempo de atraso $\left(\tau_{c}=\theta\right)$. Desta forma, apresentam-se os valores destes parâmetros e ilustram-se os desempenhos conforme a Tabela 5 e Fig. 4.

\section{A. Controlador IMC}


TABELA 5 DESEMPENHO QUANTIFICADO DO SISTEMA E OBTENÇÃO DOS PARÂMETROS PID PARA DIFERENTES VALORES DE $\tau$.

\begin{tabular}{llllll}
\hline$\tau_{C}$ & $M s$ & $I A E_{d}$ & $K_{P}$ & $K_{I}$ & $K_{D}$ \\
\hline 0,1 & 6,07 & 15,5 & 0,0077 & 8,4 & 0,008 \\
0,5 & 3,51 & 15,6 & 0,0064 & 10,0 & 0,008 \\
1 & 2,55 & 22,1 & 0,0054 & 12,0 & 0,008 \\
2 & 1,80 & 39,8 & 0,004 & 16,0 & 0,008 \\
3 & 1,62 & 62,2 & 0,0032 & 20,0 & 0,008 \\
\hline
\end{tabular}

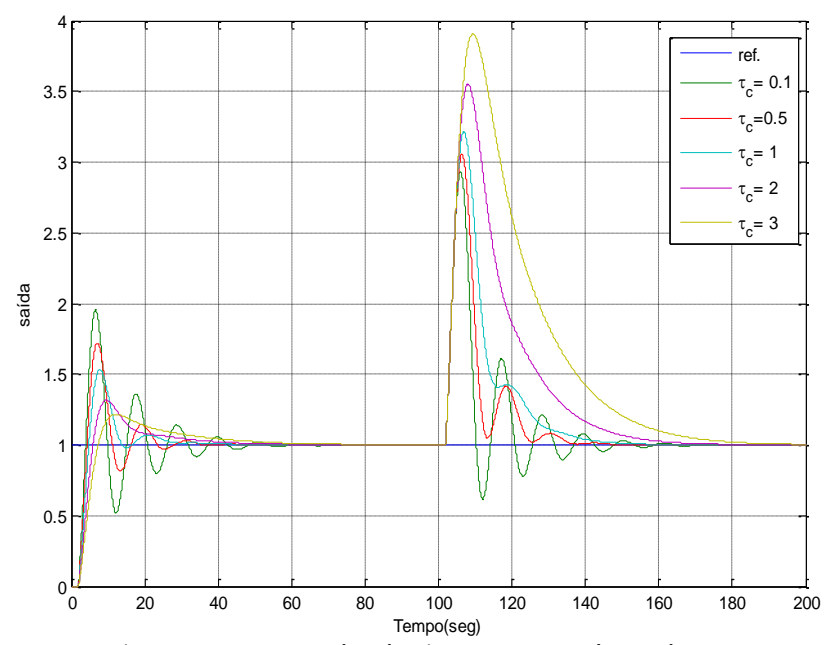

Figura 4. Desempenho do sistema para valores de $\tau_{c}$.

\section{SIMULAÇÕES E RESULTADOS}

Durante a perfuração de poços, eventualmente poderão ocorrer distúrbios que causam flutuações na pressão. Como exemplo de fontes de distúrbios podem ser citados os seguintes: o procedimento de conexão de tubos para continuar perfurando em uma maior profundidade, a perfuração em zonas geológicas de maior pressão que criam influxos, e/ou perdas de circulação, entre outros.

Nesta seção obtém-se resultados das simulações do sistema estudado e a utilização dos controladores especificados, os quais foram obtidos da linearização do modelo (Eq. 21), entretanto foram testados e simulados no processo não linear real G(s). Os controladores escolhidos e especificados levam em consideração o melhor desempenho conforme os valores de seus parâmetros. Assim, para equiparar as condições entre as duas metodologias (IMC e SIMC) escolheram-se os controladores que apresentaram similar robustez, isto é, Ms=1.8, conforme se apresenta na Tabela 6 .

TABELA 6 DESEMPENHO QUANTIFICADO E ESPECIFICAÇÃO DO CONTROLADOR PID PARA A METODOLOGIA IMC E SIMC.

\begin{tabular}{lllllll}
\hline \multirow{2}{*}{ IMC } & $\lambda$ & MS & IAEd & $K_{P}$ & $K_{I}$ & $K_{D}$ \\
\cline { 2 - 7 } & 5,5 & 1,81 & 35,1 & 0,0037 & 13,008 & 0,008 \\
\multirow{2}{*}{ SIMC } & $\tau C$ & $M s$ & IAEd & $K_{P}$ & $K_{\mathrm{I}}$ & $K_{D}$ \\
& 2 & 1,80 & 39,8 & 0,004 & 16,0 & 0,008 \\
\hline
\end{tabular}

Além do mais, estes resultados são comparados com os obtidos com um controlador Pl, o qual foi obtido e doi: https://doi.org/10.21754/tecnia.v28i2.557 especificado mediante sintonização dos seus parâmetros pelo aplicativo do Matlab, "Control System Designer". O aplicativo emprega um método de otimização do gradiente aplicado no erro da robustez e o tempo de assentamento do sistema [22]. Os valores obtidos foram: $\mathrm{KP}=0.008$ e KI $=6.15$.

\section{A. Seguimento da pressão desejada}

As técnicas de controle IMC e SIMC utilizam o modelo do sistema como referência, e assim rastrear o sinal desejado.

As primeiras simulações testam os controladores especificados para avaliar as respostas do sistema no rastreamento do sinal. As faixas de pressão de perfuração no fundo do poço podem ser muito elevadas e com grandes variações. Assim, neste trabalho se considerou uma faixa de $190-200$ bar. A Fig. 6 mostra o desempenho dos três controladores.

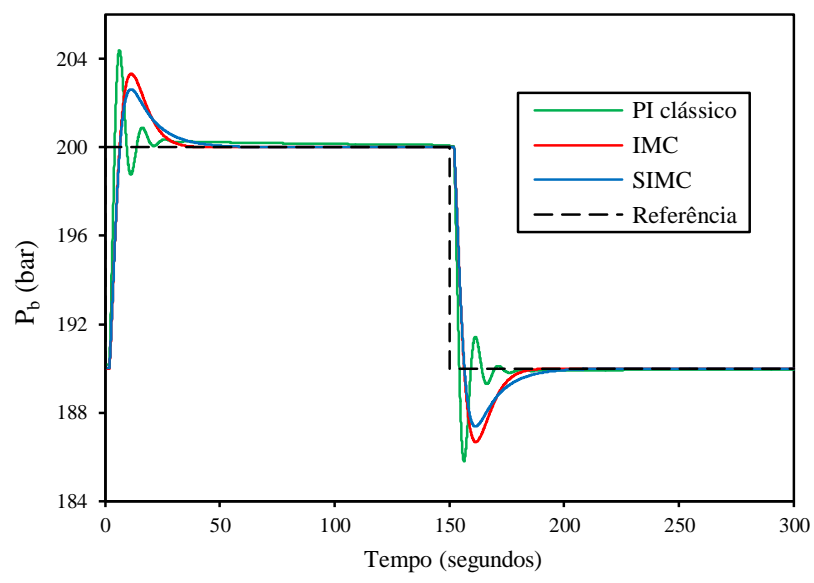

Figura 6. Resposta do sistema ao seguimento da pressão desejada.

\section{B. Conexão de tubos}

Durante este procedimento, na conexão ou adição de novos tubos na coluna de perfuração, a bomba principal de lama de vazão de $0.015 \mathrm{~m} 3 / \mathrm{s}$ deve ser desacelerada até zerar a vazão. $O$ excesso de fluido na coluna de perfuração é retirado a través de um duto, o qual volta para os tanques de lodo para reduzir a pressão da bomba de lama à pressão atmosférica. Assim, ficando só a pressão hidrostática como único elemento que influencia no comportamento da pressão anular do fundo. 


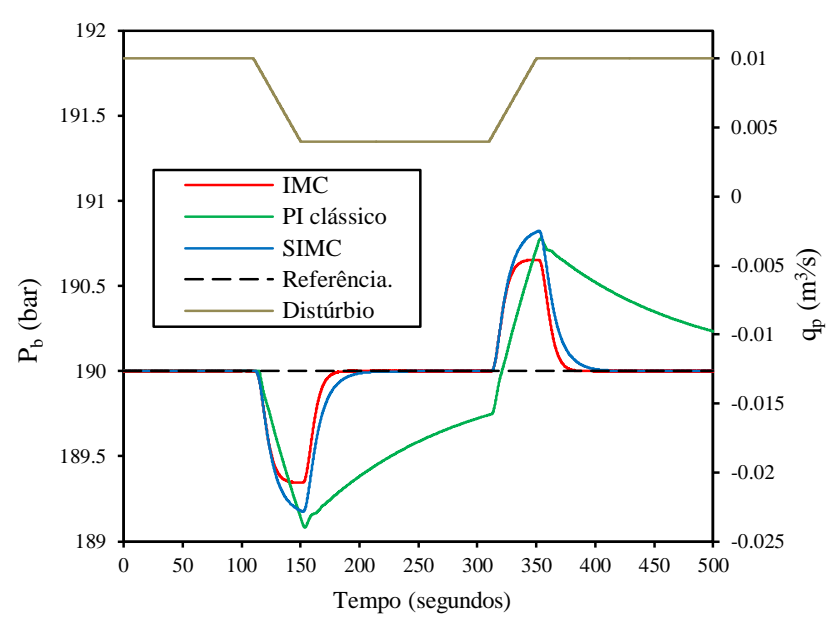

Figura 7. Resposta do sistema na conexão de tubos.

No entanto, a técnica de perfuração MPD atua ligando a bomba de contrapressão até aproximadamente $0.004 \mathrm{~m} 3 / \mathrm{s}$. Este procedimento leva aproximadamente dez minutos, para depois ligar novamente a bomba de lama [14].

Na Fig. 7 se apresenta o modelo e a resposta da simulação do distúrbio da conexão de tubulação mediante a queda de vazão na bomba principal. Utilizou-se uma função rampa de 20 até 60 segundos e no segundo 130 liga-se novamente.

\section{Influxo e/ou Kick}

Durante as operações de perfuração é comum encontrar zonas com influxo de gás ou óleo. Quando a broca entra em contato com essas regiões o fluido, seja gás u óleo, flui para o poço. Em geral, o influxo ocorre quando a pressão de formação se torna maior que a pressão induzida a partir do fluido de perfuração e o sistema de contrapressão [23].

Um influxo é produzido pelo aumento da variável de vazão do reservatório desde $0.001 \mathrm{~m}^{3} / \mathrm{s}$ até $0.02 \mathrm{~m}^{3} / \mathrm{s} \mathrm{em}$ um minuto. Na Fig. 8, para fines de simulação, o influxo pode ser considerado como uma função rampa positiva de aproximadamente 40 segundos.

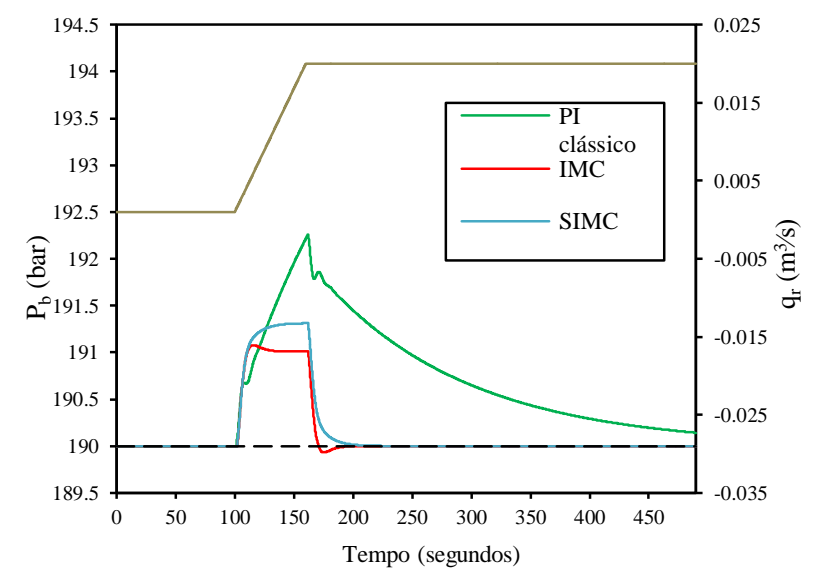

Figura 8. Resposta do sistema no influxo.
D. Perda de Lama

A perda de fluido ou lama é definida como a quantidade de lama que se filtra entre a porosidade da superfície da formação permeável que está sendo perfurada. Por causa da pressão diferencial positiva entre a pressão do poço e a pressão da formação, o fluido tende a fluir para dentro da formação [12].

A perda de lama é representada por uma queda da vazão do reservatório e que pode-se considerar o inverso do influxo, e para este caso a queda é de 0.02 $\mathrm{m} 3 / \mathrm{s}$ até $0.001 \mathrm{~m}^{3} / \mathrm{s}$. Observa-se na Fig. 9, o distúrbio simulado e a resposta dos controladores.

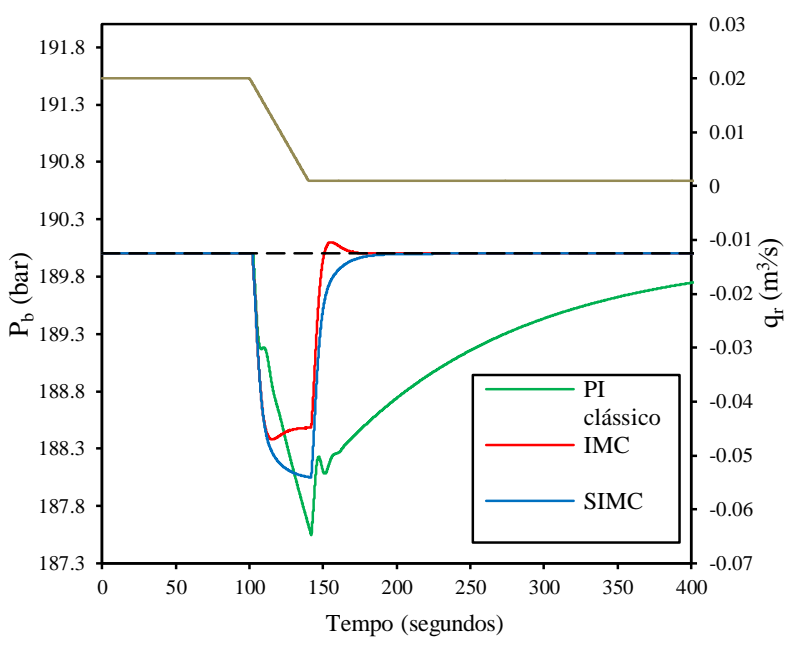

Figura 9. Resposta do sistema na perda de lama.

\section{CONCLUSÕES}

Este trabalho trata da implementação de dois tipos de metodologias de controle (IMC e SIMC) e da avaliação do comportamento e do desempenho em relação a um outro tipo de controle $\mathrm{Pl}$, sintonizado com uma outra metodologia de controle moderno. As metodologias são implementadas no controle da pressão de poços de petróleo durante a perfuração, apresenta-se o modelo com um termo integrador adicionado de um tempo de atraso no sistema. E assim, determina-se qual deles apresenta as melhores características de desempenho, sejam para obtenção do controle de pressão desejada, sejam para a presença de distúrbios.

Observam-se dos resultados das simulações, que as metodologias de controle do tipo IMC e SIMC apresentam respostas com bons comportamentos e ótimos desempenhos de ambos controladores, comparados com os resultados de um terceiro controlador.

Com relação ao controle da pressão desejada, conforme Fig. 6, observa-se que o controlador tipo SIMC apresenta melhores resultados de sobressinal em relação ao controlador IMC. No entanto, as respostas de ambos controladores em relação às perturbações (distúrbios) simuladas, sejam, devido à conexão de tubos (Fig. 7), sejam as perturbações devido à influxos 
e/ou kick (Fig. 8), e distúrbios relacionados com perdas de lama (Fig. 9), as melhores respostas e melhor desempenho são dadas pelo controlador IMC.

E finalmente, tem-se que destacar que o desempenho dos controladores IMC e SIMC, respectivamente, foram melhorados consideravelmente e testados sob condições similares aos problemas que se apresentam durante a perfuração de poços de petróleo. Acreditasse que este trabalho possa ser utilizado na comparação com outros trabalhos futuros de controle mais avançado.

\section{AGRADECIMIENTOS}

Este trabalho recebeu o financiamento do Programa de Recursos Humanos PRH48 da ANP para o setor de Petróleo e Gás

\section{REFERENCIAS}

[1] Key World Energy Statistics, International Energy Agency, 2015.

[2] O. L. Corrêa, Petróleo: Noções sobre exploração, perfuração, produção e microbiologia. Interciência, Rio de Janeiro, 2003.

[3] M. Abimbola, F. Khan e N. Khakzad, Dynamic safety risk analysis of offshore drilling. Journal of Loss Prevention in the Process Industries, vol 30, pp. 7485, 2014.

[4] B. Rehm, Manage Pressure Drilling. Gulf Publishing Company, Houston, Texas, 2008.

[5] C. M. Freitas, Acidentes de trabalho em plataformas de petróleo da Bacia de Campos, Rio de Janeiro, Brasil. Cadernos de Saúde Pública, vol. 17, no. 1, p. 117-130, 2001.

[6] M. Abimbola, Safety and risk analysis of managed pressure drilling operation using Bayesian network. Safety Science, vol. 76, pp. 133-144, 2015.

[7] I. S. Landet, Modeling and Control for Managed Pressure Drilling from Floaters. Dissertação de Mestrado - Faculty of Engineering Cybernetics, Norwegian University of Science and Technology, Norway, 2011.

[8] J-M. Godhavn, Drilling seeking automatic control solutions. IFAC-PapersOnLine, vol. 44, no. 1, pp. 10842-10850, 2011.

[9] Ø. Breyholtz, Nonlinear Model Predictive Pressure Control during Drilling Operations. Dissertação de Mestrado - Faculty of Engineering Cybernetics, Norwegian University of Science and Technology, Norway, 2008.

[10]B. J. Kennett e M. Smith, The Design of an Automated Drilling Rig: RA-D. Society of Petroleum Engineers, Amsterdam, Holanda, 1991.

[11] M. G. Folsta e A. L. Martins, Applying Theoretical Control Strategies for ROP Optimization and Offshore Well Costs Mitigation. International
Association of Drilling Conference and Exhibition. San Diego, California, 2012.

[12] J. J. Azar e G. R. Samuel, Drilling Engineering. PennWell Corporation, Tulsa, Oklahoma, 2007.

[13] H. B. Siahaan, H. Jin e F. G. Safonov, An Adaptive PID switching Controller for Pressure Regulation in Drilling. IFAC Workshop on Automatic Control in Offshore Oil and Gas. vol. 1, pp. 90-94, 2012.

[14] T. Pedersen, L1 Adaptive Control in Managed Pressure Drilling. Dissertação de Mestrado - Faculty of Engineering Cybernetics, Norwegian University of Science and Technology, Norway, 2009.

[15] J. Zhou, Adaptive Output Feedback Control of aManaged Pressure Drilling System. 47th IEE Conference on Decision and Control, Cancun México, 2008, pp. 3008-3013, 2008.

[16] G. O. Kaasa, A Simple Dynamic Model of Drilling for Control, Technical Report, StatoilHydro Resarch Centre Porsgrunn, Norway, 2006.

[17] K. Ogata, Modern Control Engineering, Ed. Pearson Education, New Jersey, 2010.

[18] D. E. Rivera, M. Morari e S. Skogestad, Internal Model Control. 4. PID Controller Design. Industrial \& Engineering Chemistry Process Design and Development, vol 25, pp. 252-265, 1986.

[19]S. Skogestad, Simple analytic rule for model reduction and PID controller tuning. Journal of Process Control, vol 14, no. 4, pp. 291-309, 2003.

[20]K. J. Åström e T. Hägglund, PID Controllers: Theory, Design and Tuning. Instrument Society of America, North Carolina, 1995.

[21] J-M. Godhavn, T. Pedersen e J. Schubert, Supervisory control for underbalanced drilling operations. IFAC -PapersOnLine, vol. 48, no. 6, pp. 120-127, 2015.

[22] H. Kwakernaak e R. Sivan, Linear Optimal Control Systems, Ed. John Wiley \& Sons, Inc., Canada, 1972.

[23] O. Gabaldon, M. Culen e P. Brand, Enhancing Well Control Through Managed Pressure Drilling. Offshore Technology Conference, Houston, Texas U.S., 2014.

Los artículos publicados por TECNIA pueden ser compartidos a través de la licencia Creative Commons: CC BY 4.0 Perú. Permisos lejos de este alcance pueden ser consultados a través del correo revistas@uni.edu.pe 